\title{
Four arms robotic-assisted pulmonary resection-left upper lobectomy: how to do it
}

\author{
Alessandro Pardolesi ${ }^{1}$, Luca Bertolaccini ${ }^{1}$, Jury Brandolini ${ }^{2}$, Piergiorgio Solli ${ }^{1}$, Pierluigi Novellis ${ }^{3}$, Giulia \\ Veronesi $^{3}$ \\ ${ }^{1}$ Department of Thoracic Surgery, Maggiore Teaching Hospital, Bologna, Italy; ${ }^{2}$ Department of Thoracic Surgery, AUSL Romagna - Forlì Teaching \\ Hospital, Forlì, Italy; ${ }^{3}$ Division of Thoracic Surgery, Humanitas Clinical and Research Centre, Rozzano, Milan, Italy \\ Correspondence to: Alessandro Pardolesi, MD. Department of Thoracic Surgery, Maggiore Hospital, Largo Nigrisoli 2, 40133 Bologna, Italy. \\ Email: a.pardolesi@gmail.com.
}

\begin{abstract}
Numerous published articles have shown the safety and efficacy of robotic anatomic pulmonary resection, including lobectomy for non-small cell lung cancer. Several techniques have been described to perform a lung lobectomy robotically. Since the beginning of our experience, we adopted a four-arm robotic approach with the da Vinci Si System. More recently we have used the newer Xi model, that offers a simplified and "quicker" set-up and docking time. This article emphases specifically on the technical aspects of how to complete the hilar dissection during four-arm robotic lobectomy.
\end{abstract}

Keywords: Robot-assisted; lung cancer; lobectomy

Received: 04 January 2018; Accepted: 27 April 2018; Published: 22 May 2018.

doi: 10.21037 /jovs.2018.05.04

View this article at: http://dx.doi.org/10.21037/jovs.2018.05.04

\section{Introduction}

Many retrospective studies have demonstrated that robotassisted lung lobectomy is feasible and safe, and initial longterm results indicate that the oncological outcome is like that reported for open and video-assisted thoracoscopic surgery (VATS) approaches. However, randomised controlled trials are still missing (1).

Different approaches have been described for performing a lung lobectomy robotically. The robotic-assisted thoracoscopic surgery (RATS) introduced by Dr. Bernard Park [2006] and the complete portal robotic lobectomy (CPRL) described by Cerfolio [2014] and Dylewski [2011] are the two principal techniques reported in the literature (2-4). The differences are related to the position of the utility incision and the operative ports, insufflation with $\mathrm{CO}_{2}$ and the type of approach to the hilar structures.

Since the beginning of our experience, we adopted a modified RATS technique as described by Veronesi et al. $(5,6)$. Our approach is based on performing a 3 to $4 \mathrm{~cm}$ utility incision (no rib spread) at the fifth intercostal space anteriorly, an $8 \mathrm{~mm}$ camera port and two more $8-10 \mathrm{~mm}$ operative ports. We do not routinely use the $\mathrm{CO}_{2}$ insufflation as we have not found many advantages, the utility incision offers a spare port for the bed assistant to introduce instruments and pull out the surgical specimen at the end of the procedure.

We initially used the da Vinci Si System, and more recently we have been using the newer model Xi System without modifying the surgical approach. This article focuses specifically on the technical aspects of how to complete the hilar dissection during four-arm robotic left upper lobectomy.

\section{Clinical vignette}

The patient was a 58-year-old gentleman, smoker (30 pack years), with no significant medical history, who received radiological follow-up for a previous pulmonary inflammation. Chest X-ray revealed the presence of a spiculated opacity in the left lung. The Chest Computer Tomography and PET scan detected a $15 \mathrm{~mm} \times 12 \mathrm{~mm}$ solid lesion located in the upper lobe of the left lung with a standardized uptake value (SUV) of 6 (Figure 1A,B). The transthoracic fine needle biopsy confirmed the malignant 

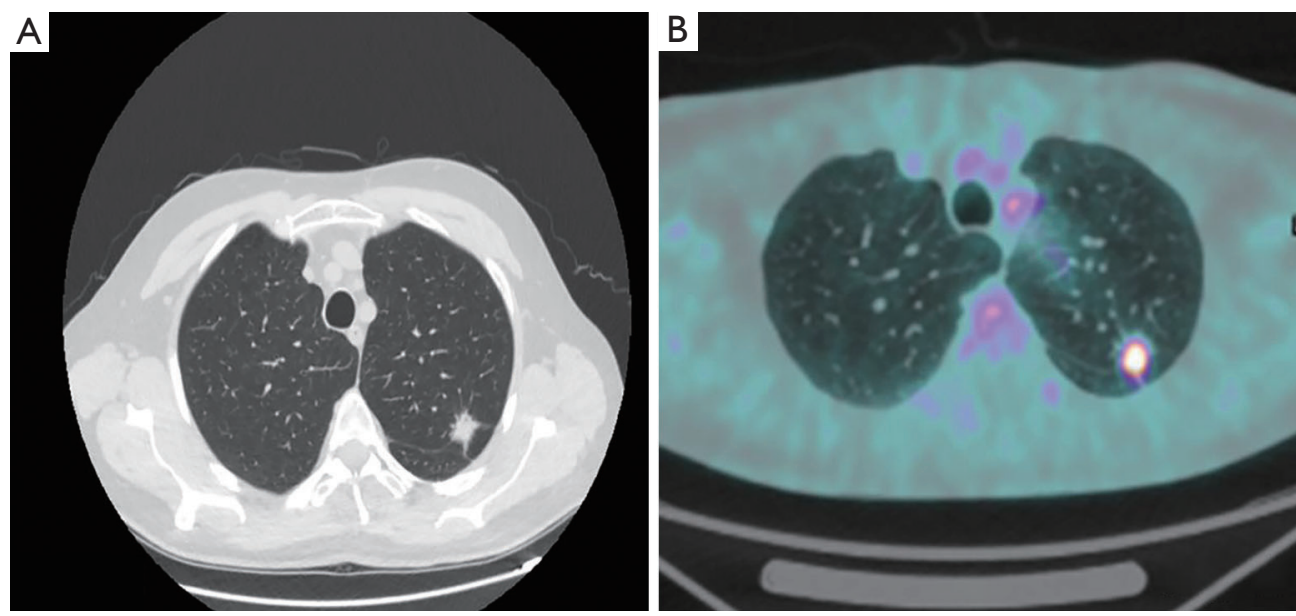

Figure 1 (A,B) Adenocarcinoma of the left upper lobe. Standardized uptake value (SUV) 6 with PET scan.

nature of the lesion. The final preoperative stage was T1aN0 adenocarcinoma. The patient was scheduled for a four-arm left upper robotic lobectomy. The overall operative time was 125 minutes. The patient was discharged on the third postoperative day. The procedure was performed with four arms robotic approach the da Vinci Xi Surgical System.

\section{Preference cards}

(I) 3D high-definition camera (30 lens; $8 \mathrm{~mm})$;

(II) One Fenestrated Bipolar Forceps: these forceps allow dissection and coagulation and are very useful to speed-up the procedure;

(III) Tip-Up fenestrated grasper: atraumatic forceps designed for the lung, which is used for retracting parenchyma and exposing the hilum;

(IV) Curved Bipolar Dissector: compared to the permanent cautery hook offer safer dissection of vessels and structures surrounding the hilum with no need of a second instrument to encircle the vessels;

(V) Endo GIA ${ }^{\mathrm{TM}} 30 \mathrm{~mm}$ Curved Tip Articulating Vascular/ Medium Reload with Tri-Staple ${ }^{\mathrm{TM}}$ Technology (Covidien);

(VI) Endowrist $8 \mathrm{~mm}$ clip appliers (medium-large clips): it is recommended for dissection of small pulmonary artery (AP) branches (up to $8 \mathrm{~mm}$ );

(VII) Energy device: Harmonic ACE ultrasonic;

(VIII) Alexis ${ }^{\mathrm{TM}}$ (Applied Medical) soft tissue retractor;

(IX) Vessel loops.

\section{Surgical technique}

\section{Patient positioning and port placement}

The patient is placed in right lateral decubitus with the hips fixed at the level of the table break.

We place the Xi da Vinci at the back of the patient. The $\mathrm{Xi}$ has a rotating system connected to the four arms that simplify the set-up and docking time.

We proceed to perform a $3-\mathrm{cm}$ utility incision at the fifth intercostal space anteriorly of the latissimus dorsi, With the $30^{\circ}$ camera we explore the chest cavity to confirm the absence of pleural effusion and metastasis. Under view guidance, we place the remaining ports. The $8-\mathrm{mm}$ camera port is placed at the level of the mid-axillary line at the seventh intercostal space, and two $(8 \mathrm{~mm})$ operative ports are performed in the eighth intercostal space and the auscultatory triangle, respectively. We then complete the set-up docking the Robot. The bed-assistant introduce the operative robotics arms. The Tip- $\mathrm{Up}$ fenestrated grasper is inserted through the fourth posterior trocar, and it is used to retract the lung parenchyma exposing the lung hilum. The fenestrated bipolar forceps is then placed at the utility incision port, and the curved bipolar dissector is finally introduced through the operative port (Figure 2).

We report our technique to perform a robotic left upper lobectomy with an anterior fissureless approach.

Surgical sequence: (I) left upper pulmonary vein; (II) AP truncus anterior branch; (III) left upper lobe bronchus; (IV) Lingula branch and posterior ascending AP branches; (V) lymph node dissection (Figure 3). 


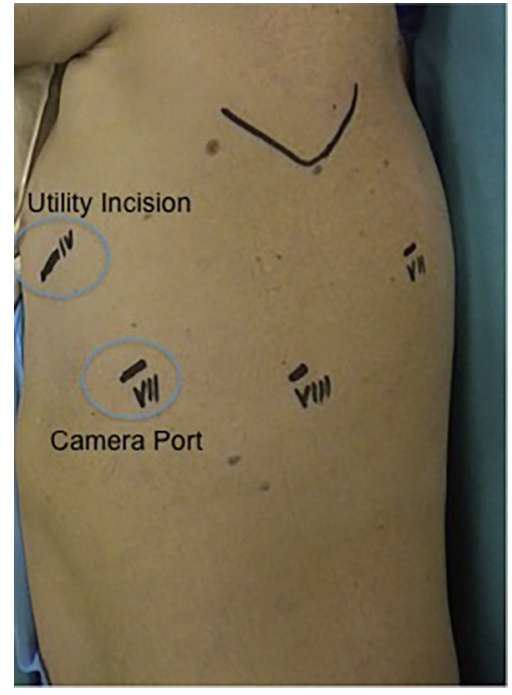

Figure 2 Right lateral decubitus port placement. The camera port is usually placed in the seventh intercostal space more laterally compared to the right-sided lobectomy so to keep the hart out of the view.

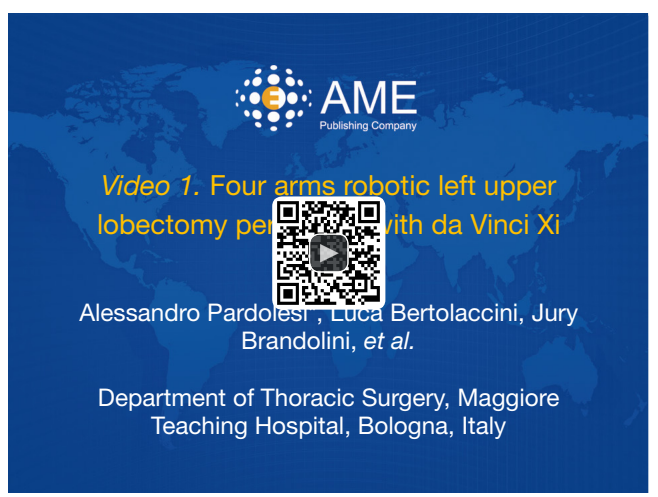

Figure 3 Four arms robotic left upper lobectomy performed with da Vinci Xi System (7).

Available online: http://www.asvide.com/article/view/24891

\section{Step 1}

As for the right upper lobectomy we approach the hilum isolating first the upper lobe vein. We use the Tip-Up grasper to retract the lobe posteriorly and expose the hilum. The mediastinal pleura over the vein is opened. The bifurcation between the upper and lower vein should be dissected at this time. This manoeuvre should help to isolate the vein from the inferior board. To safely surround the vein, the fascia over it should be dissected with a combination of blunt and sharp dissection. The use of

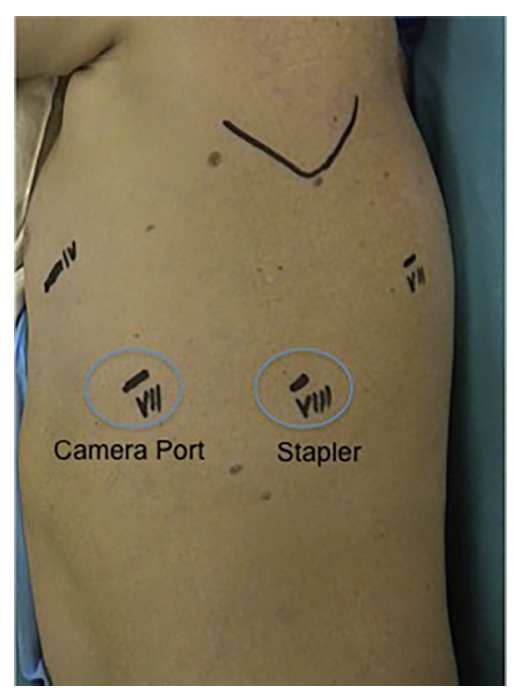

Figure 4 To perform a robotic left upper lobectomy we introduce the stapler always from the posterior port as shown in the figure.

a vessel loop to encircle the structure is not mandatory. However, it may help in some circumstances if the angulation of the staler is not straightforward and the tip of the stapler engage the main AP. The stapler is introduced form the posterior port as shown in Figure 4.

\section{Step 2}

Once the vein is divided our preference is to have the PA anterior trunk divided first to facilitate isolation and division of the upper lobe. The lobe is retracted posteriorly and towards the diaphragm. The mediastinal pleura is dissected around the hilum proceeding form the divided vein upward around the AP extending the dissection posteriorly. The AP truncus is dissected using sharp and blunt dissection in the same manner as with the pulmonary vein. The lobe should be now retracted towards the apex and slightly anteriorly to open the space behind the artery and enlarge the pathway to advance the stapler around the vessel safely. The Vascular stapler is introduced from the posterior port (Figure 4).

\section{Step 3}

Once the PA anterior trunk is divided the lobe should be pulled more anteriorly and upward to facilitate the identification of the left upper bronchus that arises vertically from the main bronchus. Isolation of the bronchus should start from the inferior edge. Once the lingula branch is identified, the lymph node that lies between the bronchus 
and the artery should be dissected away leaving a larger space for instruments to tackle the inferior board and proceed behind it. Isolation of the left upper bronchus must be performed with extreme caution. The Cadiere forceps should be kept in contact with the back wall of the bronchus to avoid injuries of the interlobar AP. The stapler is again introduced from the posterior port.

\section{Step 4}

The lobe is now pulled posteriorly and towards the diaphragm. The lingual artery and the posterior ascending branches are then isolated and dissected using sharp and blunt dissection in the same manner as for the vein and the anterior AP trunk. We prefer dividing the lingual artery first, and we approach the posterior branches afterwards.

At this stage, the lobectomy is completed by dividing the upper lobe from the lower lobe according to the fissureless (or fissure-last) technique. The resected lobe is positioned inside a big specimen and retrieved out of the utility incision.

\section{Step 5}

For all cases of malignancy, systemic lymph node dissection is mandatory. For any left-sided robotic lobectomy, we routinely dissect stations 5, 7 L8-9 and station 7 .

Stations 8 and 9 are removed during incision of the pulmonary ligament. The sub-carinal area is approached retracting the remaining lobe anteriorly towards the utility incision to have better exposure of the target area. The mediastinal pleura is dissected from the superior edge of the lower lobe vein all the way up along the course of the vagus nerve. Extensive dissection of the posterior mediastinal pleura affords a clear visualisation of the oesophagus and the main left bronchus.

Stations 5 and 6 are dissected out by opening the mediastinal pleura along the course of the phrenic nerve upwards to expose the aortic arch.

\section{Tips/discussion points}

(I) Having a utility incision from the start of surgery affords more security in the necessity of immediate conversion for a vascular accident. It is used as a window through which the surgical specimen can be removed, and when required, can be used for wedge resections before the lobectomy, and or finger palpation of small nodules.

(II) The technique here defined is a "fissure last". If the fissure is complete, dissection of the lingula artery and posterior ascending branches can be performed before stapling the fissure after the upper bronchus. The posterior AP is separate proceeding with a posterior approach to the hilum. Even in this situation, the stapler should be introduced from the posterior port as shown in Figure 4.

(III) Small PA branches should be considered for the Endowrist clip appliers. Posterior ascending branches can be multiple (1 to 4 ) and short with a small calibre. We usually place one or two clips on the proximal end, and we divide the vessels with the bipolar energy.

(IV) To perform a four-arm robotic lobectomy, we introduce the stapler from the same posterior port for all the four steps described above.

\section{Conclusions}

The four arms robotic approach is practicable, reproducible and appears to be well suited to perform major lung resections safely. In the last few years, robotic lobectomy has been progressively adopted by many general thoracic surgeons for lung resection. Furthermore, robotic technology is evolving, and thoracic surgeons should critically evaluate this evolution because, as stated from Leonardo da Vinci who gave his name to the surgical robot: "Those who fall in love with science without practice are like the helmsman who goes into a boat without a rudder or a compass and has no assurance of where to go".

\section{Acknowledgements}

None.

\section{Footnote}

Conflicts of Interest: The authors have no conflicts of interest to declare.

Informed Consent: Written informed consent was obtained from the patient for publication of this manuscript and any accompanying images.

\section{References}

1. Kent M, Wang T, Whyte R, et al. Open, video-assisted 
thoracic surgery, and robotic lobectomy: review of a national database. Ann Thorac Surg 2014;97:236-42; discussion 242-4.

2. Park BJ, Flores RM, Rusch VW. Robotic assistance for video-assisted thoracic surgical lobectomy: technique and initial results. J Thorac Cardiovasc Surg 2006;131:54-9.

3. Cerfolio RJ. Total port approach for robotic lobectomy. Thorac Surg Clin 2014;24:151-6, v.

4. Dylewski MR, Ohaeto AC, Pereira JF. Pulmonary resection using a total endoscopic robotic video-assisted approach. Semin Thorac Cardiovasc Surg 2011;23:36-42.

doi: 10.21037 /jovs.2018.05.04

Cite this article as: Pardolesi A, Bertolaccini L, Brandolini J, Solli P, Novellis P, Veronesi G. Four arms robotic-assisted pulmonary resection-left upper lobectomy: how to do it. J Vis Surg 2018;4:109.
5. Veronesi G, Galetta D, Maisonneuve P, et al. Four-arm robotic lobectomy for the treatment of early-stage lung cancer. J Thorac Cardiovasc Surg 2010;140:19-25.

6. Pardolesi A, Bertolaccini L, Brandolini J, et al. Four arms robotic-assisted pulmonary resection-left lower lobectomy: how to do it. J Thorac Dis 2017;9:1658-62.

7. Pardolesi A, Bertolaccini L, Brandolini J, et al. Four arms robotic left upper lobectomy performed with da Vinci Xi System. Asvide 2018;5:517. Available online: http://www. asvide.com/article/view/24891 